Pacific

Journal of

Mathematics

INJECTIVITY RADII OF HYPERBOLIC POLYHEDRA

Joseph D. Masters

Volume $197 \quad$ No. 2

February 2001 


\title{
INJECTIVITY RADII OF HYPERBOLIC POLYHEDRA
}

\author{
Joseph D. MASTERS
}

\begin{abstract}
We define the injectivity radius of a Coxeter polyhedron in $\mathrm{H}^{3}$ to be half the shortest translation length among hyperbolic/loxodromic elements in the orientation-preserving reflection group. We show that, for finite-volume polyhedra, this number is always less than $2.6339 . .$. , and for compact polyhedra it is always less than $2.1225 . .$.
\end{abstract}

\section{Introduction.}

Hyperbolic reflection groups are discrete groups of isometries of hyperbolic space generated by reflections in the faces of a polyhedron. They provided some of the earliest known examples of Kleinian groups, and have been wellstudied (see $[\mathbf{V}]$ ). In this paper, we prove that 3-dimensional hyperbolic reflection groups always contain short elements.

To be more precise, let $\Gamma^{+}(P)$ be the group of orientation-preserving isometries generated by reflections in the faces of the polyhedron $P$. We define injrad $\left(\Gamma^{+}(P)\right)$ to be half the shortest translation length among hyperbolic/loxodromic elements of $\Gamma^{+}(P)$. Then we have:

Theorem 4.1 (Main Theorem). Let $P$ be a finite-volume Coxeter polyhedron in $\mathbf{H}^{3}$. Then injrad $\left(\Gamma^{+}(P)\right)<\cosh ^{-1}(7)=2.6639 \ldots$. If $P$ is compact, then injrad $\left(\Gamma^{+}(P)\right)<\cosh ^{-1}(3+4 \cos (2 \pi / 5))=2.1225 \ldots$.

\section{Remarks.}

1. It is known (see $[\mathbf{W}]$ ) that if $\left\{\mathbf{H}^{3} / \Gamma_{i}\right\}$ is a family of closed hyperbolic 3 -manifolds, and if $\left\{\operatorname{rank}\left(\Gamma_{i}\right)\right\}$ is bounded, then $\left\{\operatorname{injrad}\left(\mathbf{H}^{3} / \Gamma_{i}\right)\right\}$ is also bounded (recall the rank of a finitely generated group is the cardinality of a minimal generating set). Observe that by $[\mathbf{B}]$, $\operatorname{Rank}(\Gamma(P))$ increases with the number of sides of $P$, so Theorem 4.1 is not covered by $[\mathbf{W}]$.

2. For more about short geodesics in hyperbolic 3-manifolds, see $[\mathbf{A R}]$.

3. We speculate that the bounds may be sharp, but we do not know a proof.

Idea of Proof. To prove the Main Theorem, we must show that every three-dimensional hyperbolic reflection group contains a hyperbolic element 
with suitably short translation length. This can usually be done by finding two non-adjacent faces of the polyhedron which are suitably close; the short element is obtained by composing the reflections in the corresponding hyperplanes.

A result of Nikulin's guarantees that every Coxeter polygon which is not a triangle has two non-adjacent sides which are close, and a two-dimensional version of the Main Theorem follows easily. For most polyhedra, we can use Nikulin's result to show that two non-adjacent faces are close; the exceptions are those which contain "non-prismatic" faces (see Section 2 for a definition). We show that these exceptional cases always contain triangular faces. Then, after extending to the sphere at infinity, we use combinatorics and Euclidean geometry to deduce the existence of a short element. The results on hyperbolic polyhedra contained in Section 3 allow us to sharpen the bound.

Organization. Section 2 contains basic definitions; Section 3 contains some general, technical results about hyperbolic polyhedra; Section 4 contains the proof of the Main Theorem.

Acknowledgments. I would like to thank Alan Reid for his help and encouragement. Thanks also to Gary Hamrick and David Bachman for valuable conversations, Daryl Cooper for helpful correspondence, and the referee for valuable comments.

\section{Definitions.}

A convex polyhedron, $P$, in $\mathbf{H}^{n}$ is a countable intersection of closed halfspaces: $P=\bigcap_{i} H_{i}^{+}$, where $H_{i}$ denotes a hyperplane and $H_{i}^{+}$the corresponding closed half-space. When $\mathrm{n}=2$, we use the term polygon instead. If $P$ is a convex polyhedron in $\mathbf{H}^{n}$, we let $\Gamma(P)$ denote the group of isometries generated by reflections in the bounding hyperplanes of $P . \Gamma^{+}(P)$ denotes its orientation-preserving subgroup of index 2 . We say that a finite-volume, convex polyhedron $P$ is a Coxeter polyhedron if its dihedral angles are all integer submultiples of $\pi$; if $P$ is a Coxeter polyhedron, then $\Gamma^{+}(P)$ is discrete. Two faces of $P$ are adjacent if they share an edge. Given a hyperplane $H$ in $\mathbf{H}^{n}, \rho_{H}$ will denote the isometry obtained by reflection in $H$. We will denote the hyperbolic distance between two sets $X$ and $Y$ in $\mathbf{H}^{n}$ by $d(X, Y)$.

Given an n-sided face $F$ of $P(n>3)$, label the edges of $F$ by $E_{1}, \ldots, E_{n}$, where $E_{i}$ shares a vertex with $E_{i+1}$ for $i=1,2, \ldots, n-1$, and label the adjacent faces by $F_{1}, \ldots, F_{n}$, where $F$ and $F_{i}$ share edge $E_{i}$ (we say the faces adjacent to $F$ are labeled "cyclicly"). We say that $F$ is prismatic if, for $i, j=1, \ldots, n,|i-j|>1(\bmod n)$ implies $F_{i}$ is non-adjacent to $F_{j}$.

By a hyperbolic $n$-manifold, $\mathbf{H}^{n} / \Gamma$, we mean the quotient of hyperbolic $\mathrm{n}$-space by a discrete group of isometries acting freely. If $\Gamma$ has torsion, the quotient space $H^{n} / \Gamma$ is a hyperbolic $n$-orbifold. The injectivity radius 
of a hyperbolic manifold $M=\mathbf{H}^{n} / \Gamma$ is equal to $\sup \left\{\alpha \in \mathbb{R}^{+} \mid\right.$every point $x \in M$ is the center of an embedded ball of radius $\alpha\}$. We shall generalize this definition to the case where $\Gamma$ has torsion: The injectivity radius of a Kleinian group $\Gamma$, denoted injrad $(\Gamma)$, is equal to half the shortest translation length among hyperbolic/loxodromic elements of $\Gamma$. Note that this agrees with the usual notion when $\Gamma$ is torsion-free. The injectivity radius of a Coxeter polyhedron $P$ is equal to injrad $\left(\Gamma^{+}(P)\right)$. Given an element $g$ of $\Gamma$, we will denote its translation length by $\ell(g)$.

\section{Hyperbolic Polyhedra.}

The following technical result shall be used in our proof of the Main Theorem.

Theorem 3.1. Let $P$ be a finite-volume, convex polyhedron in $\boldsymbol{H}^{3}$ with acute dihedral angles and no triangular faces. Then $P$ has a prismatic face. If, furthermore, $P$ is compact, then $P$ has a prismatic quadrilateral or pentagonal face.

Proof. We first consider the finite-volume case.

Lemma 3.2. Let $P$ be a finite-volume, convex polyhedron in $\boldsymbol{H}^{3}$ with acute dihedral angles and no triangular faces, and suppose that $P$ contains at least one non-prismatic face. Let $G$ be a planar graph representing the 1-skeleton of $P$ (see Fig. 1). Then there are three non-prismatic faces of $P$ which bound a region in $G$ consisting entirely of prismatic faces.

Proof. Let $F$ be a non-prismatic face of $\mathrm{P}$, and label its adjacent faces cyclicly by $F_{1}, F_{2}, \ldots$ We have that $F_{i}$ and $F_{j}$ are adjacent for some $i, j$ with $|i-j|>1$ (see Fig. 1). Note that $F, F_{i}$, and $F_{j}$ bound a region $R$, and that $F_{i}$ and $F_{j}$ are also non-prismatic. Since $P$ contains no triangular faces, $R$ cannot be a face. And if the lemma is false, $R$ must contain a non-prismatic face $F^{\prime}$. Label the faces adjacent to $F^{\prime}$ cyclicly by $F_{1}^{\prime}, F_{2}^{\prime}, \ldots$ Then for some $k, \ell$ with $|k-\ell|>1, F_{k}^{\prime}$ and $F_{\ell}^{\prime}$ are adjacent. Then $F^{\prime}, F_{k}^{\prime}$, and $F_{\ell}^{\prime}$ bound a triangular region $R^{\prime} \subsetneq R$. Again, if the lemma is false, $R^{\prime}$ must contain a non-prismatic face $F^{\prime \prime}$, creating a triangular region $R^{\prime \prime} \subsetneq R^{\prime}$. Since $P$ has a finite number of sides, this process must eventually terminate with three non-prismatic faces bounding a region containing only prismatic faces. This proves the lemma, and the non-compact case of Theorem 3.1 follows immediately.

To prove the stronger statement for the compact case, we shall need the following combinatorial lemma:

Lemma 3.3. Let $P$ be a compact, convex polyhedron in $\boldsymbol{H}^{3}$ with acute dihedral angles. Then $P$ contains a face with 5 or fewer edges. 


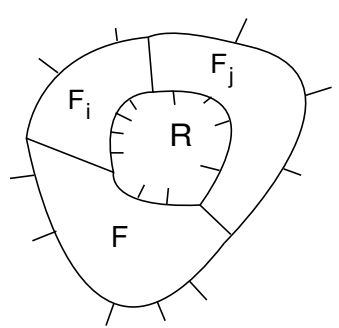

Graph G

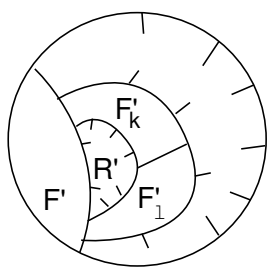

Graph G'

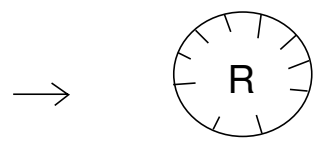

Graph G'

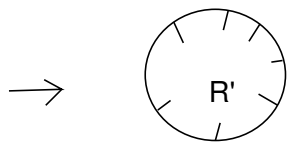

Graph G"

Figure 1. Reducing the graph around a non-prismatic face.

Proof. This follows from an Euler characteristic count. Let $|V|=$ number of vertices of $P,|E|=$ number of edges of $P$, and $|F|=$ number of faces of $P$. Since $P$ is a compact, convex hyperbolic polyhedron, $P$ is simple- i.e., each vertex is shared by exactly 3 different edges (see $[\mathbf{A} 1]$ ). So $|V|=2|E| / 3$.

We have $|V|-|E|+|F|=2$.

$|F|-|E| / 3=2$.

$|F|(1-|E| / 3|F|)=2$.

So $|E| /|F|<3$.

So the average number of edges per face $<6$. So $P$ must contain a face with 5 or fewer edges.

Now suppose $P$ is compact. If all faces of $P$ are prismatic, we are done by Lemma 3.3. So suppose $P$ contains a non-prismatic face. Then by Lemma 3.2 , there are three non-prismatic faces of $P$ which bound a region $R$ in $G$ consisting entirely of prismatic faces. We need to show that $R$ contains a face with 5 or fewer edges. We form $\hat{R}$ from $R$ by subtracting the three vertices, $v_{1}, v_{2}, v_{3}$, of non-prismatic faces on the boundary of $R$, and then adding a face to the exterior of $R$, so that $\hat{R}$ is topologically a sphere (see Fig. 2).

Then, arguing as in Lemma 3.3, $\hat{R}$ must contain a face $F$ with 5 or fewer edges. We claim that $R$ must also contain such a face. This will certainly be true unless 

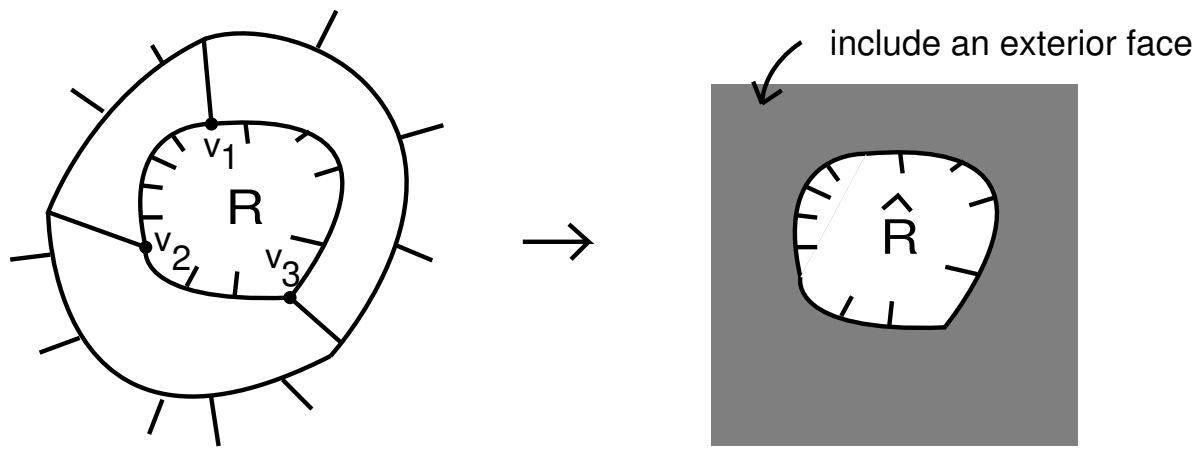

Figure 2. To form $\hat{R}$ from $R$, we remove three vertices and include an exterior face.

1. $F$ is the face on the exterior of $R$.

Or

2. $F$ is a pentagon, and $F$ contains one of the edges from which a vertex was deleted (note that $F$ can contain at most one of the $v_{i}$ 's, since it is prismatic).

If $F$ is the face on the exterior and $F$ is a triangle, then we claim that $R$ must consist of three quadrilaterals, as in Fig. 3b. For otherwise, $R$ must contain three non-prismatic faces, contrary to assumption (see Fig. 3b).

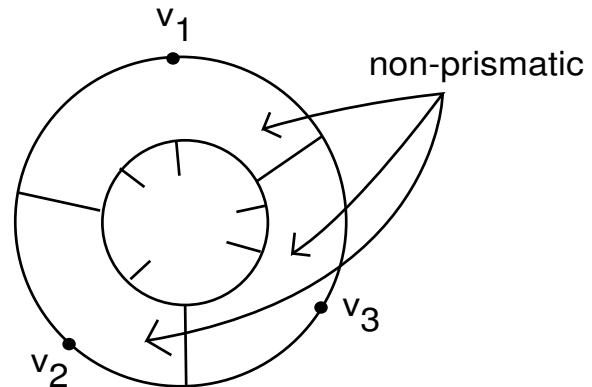

a

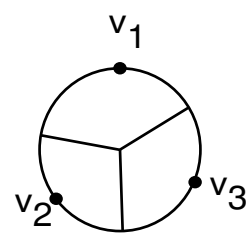

b

Figure 3. If the exterior is a triangle, then $R$ will contain either a) non-prismatic faces, or b) three quadrilaterals. 
So if the theorem is false, then $\hat{R}$ contains at most four faces with fewer than 6 sides; at least three of them must be pentagons, and none are triangles. We shall show that this is impossible.

Number the faces of $\hat{R}$ by $F_{1}, F_{2}, \ldots, F_{n}$. Let $\left|E_{j}\right|$ be the number of sides of $F_{j}$. Then $\left|E_{1}\right|+\cdots+\left|E_{n}\right|=2|F|$, where $|F|$ is the total number of faces of $\hat{R}$. Since $P$ is simple, the graph is trivalent, so $|F|$ is divisible by 3 ; hence $\left|E_{1}\right|+\cdots+\left|E_{n}\right|$ is divisible by 6 . Since also the average number of sides per face is $<6$, we have:

$$
6 n-5 \leq \sum_{i=1, \ldots, n}\left|E_{i}\right|<6 n .
$$

Thus the sum is not divisible by 6 , for a contradiction.

\section{Injectivity radius of hyperbolic polyhedra.}

In this section we prove the Main Theorem:

Theorem 4.1. Let $P$ be a finite-volume Coxeter polyhedron in $\boldsymbol{H}^{3}$. Then injrad $\left(\Gamma^{+}(P)\right)<\cosh ^{-1}(7)=2.6339 \ldots$. If $P$ is compact, then injrad $\left(\Gamma^{+}(P)\right)<\cosh ^{-1}(3+4 \cos (2 \pi / 5))=2.1226 \ldots$.

An important part of the proof of Theorem 4.1 is played by the following 2-dimensional result.

Theorem 4.2. Let $P$ be a finite-area Coxeter n-gon in $\boldsymbol{H}^{2}, n>3$. Then injrad $\left(\Gamma^{+}(P)\right) \leq \cosh ^{-1}(3+4 \cos (2 \pi / n))<\cosh ^{-1}(7)=2.6339 \ldots$. If $n=3$, then injrad $\left(\Gamma^{+}(P)\right) \leq \cosh ^{-1}(3+4 \cos (2 \pi / 4))$. If $P$ is compact, then all the inequalities are strict.

Proof. The theorem is a consequence of the following lemma, which is a re-phrasing of ([N, Theorem 3.2.1]).

Lemma 4.3. Let $P$ be a finite area, convex n-gon in $\boldsymbol{H}^{2}$. Label the bounding geodesics of $P$ cyclicly by $H_{1}, H_{2}, \ldots, H_{n}$. Then for some $i, d\left(H_{i}, H_{i+2}\right) \leq$ $\cosh ^{-1}(3+4 \cos (2 \pi / n))$ (subscripts taken $\left.\bmod n\right)$. If $P$ is compact, the inequality is strict.

Proof. We will reproduce Nikulin's proof that $d\left(H_{i}, H_{i+2}\right)<\cosh ^{-1}(7)$. The proof of the finer estimate is a bit more complicated, and we omit it (see $[\mathbf{N}]$ ).

We use the Lobachevsky model for $\mathbf{H}^{2}$. First, pick a point $p$ on the interior of $P$. Project the vertices of $P$ onto the circle at infinity along rays emanating from $p$. This determines an ideal polygon $P^{\prime}$ with bounding geodesics $H_{1}^{\prime}, H_{2}^{\prime}, \ldots$ We claim that $d\left(H_{i}, H_{i+2}\right) \leq d\left(H_{i}^{\prime}, H_{i+2}^{\prime}\right)$ : The distance between $H_{i}^{\prime}$ and $H_{i+2}^{\prime}$ is measured along a mutually orthogonal geodesic segment $\alpha$ (see Fig. 4a); since $\alpha$ must intersect $H_{i}$ and $H_{i+2}$, $d\left(H_{i}, H_{i+2}\right)<\operatorname{length}(\alpha)=d\left(H_{i}^{\prime}, H_{i+2}^{\prime}\right)$. 


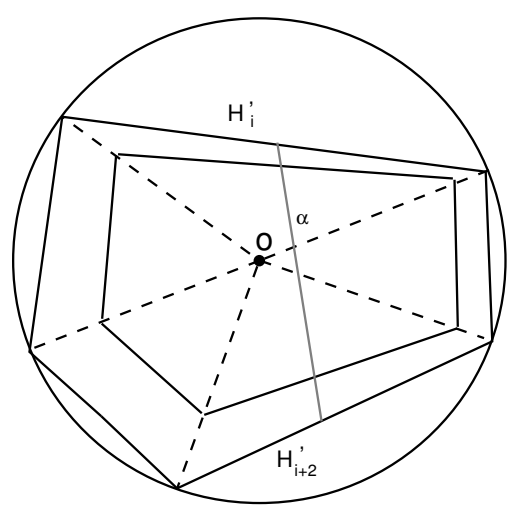

a

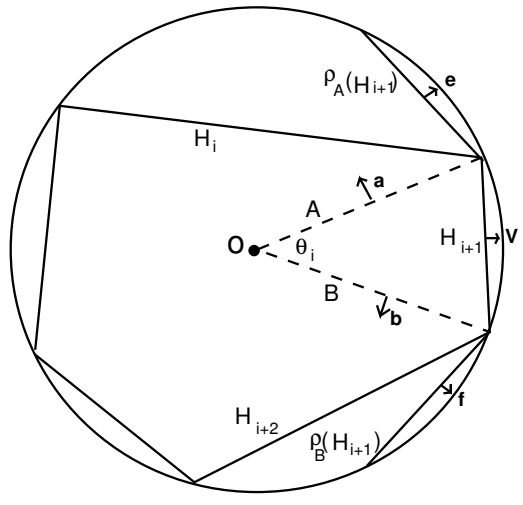

b

Figure 4. a) The faces of the ideal polygon are farther apart.

b) Notation for Lemma 3.2.

So it is enough to consider the case where $P$ is an ideal polygon. Pick $i$ such that the Euclidean angle $\theta_{i}=v_{i} O v_{i+1}$ is minimal, where $O$ is the origin. Let $A$ be the diameter through $v_{i}$ and let $B$ be the diameter through $v_{i+1}$. Then $d\left(H_{i}, H_{i+2}\right) \leq d\left(\rho_{A}\left(H_{i+1}\right), \rho_{B}\left(H_{i+1}\right)\right)=\cosh ^{-1}(-\langle\mathbf{e}, \mathbf{f}\rangle)$, where $\mathbf{e}$ is the unit normal to $\rho_{A}\left(H_{i+1}\right), \mathbf{f}$ is the unit normal to $\rho_{B}\left(H_{i+1}\right)$, and $\langle$.$\left.| . \right\rangle$ is the inner product $\left\langle\left(x_{1}, y_{1}, z_{1}\right) \mid\left(x_{2}, y_{2}, z_{2}\right)\right\rangle=x_{1} x_{2}+y_{1} y_{2}-z_{1} z_{2}$ (see Fig. $4 b)$.

Now, let $\mathbf{v}, \mathbf{a}$ and $\mathbf{b}$ be outward unit normals to $H_{i+1}, A$ and $B$, respectively. Then we have:

$$
\begin{aligned}
\langle\mathbf{e}, \mathbf{f}\rangle & =\langle\mathbf{v}-\mathbf{2}\langle\mathbf{v}, \mathbf{a}\rangle \mathbf{a}, \mathbf{v}-\mathbf{2}\langle\mathbf{v}, \mathbf{b}\rangle \mathbf{b}\rangle \\
& =\langle\mathbf{v}, \mathbf{v}\rangle-\mathbf{2}\langle\mathbf{v}, \mathbf{a}\rangle^{\mathbf{2}}-\mathbf{2}\langle\mathbf{v}, \mathbf{b}\rangle^{2}+\mathbf{4}\langle\mathbf{v}, \mathbf{a}\rangle\langle\mathbf{v}, \mathbf{b}\rangle\langle\mathbf{a}, \mathbf{b}\rangle \\
& =1-2-2-4 \cos \left(\theta_{i}\right) .
\end{aligned}
$$

So $d\left(H_{i}, H_{i+2}\right)<\cosh ^{-1}(7)$.

We now resume the proof of Theorem 4.2. Let $P$ be a Coxeter n-gon, and suppose first $n>3$. Pick two non-adjacent edges such that the corresponding geodesics $H$ and $H^{\prime}$ are less than $\cosh ^{-1}(3+4 \cos (2 \pi / n))$ apart. Let $g=\rho_{H} \rho_{H^{\prime}}$ in $\Gamma^{+}(P)$. Since $P$ has acute angles, $H$ is disjoint from $H^{\prime}$, so $g$ is hyperbolic. Let $\alpha$ denote the axis of $g$, and note that it is perpendicular to both $H_{i}$ and $H_{i+2}$ (see Fig. 5). $\ell(g)$ is given by $d(p, g(p))$, where $p$ is any point on $\alpha$. Taking $p$ to be $\alpha \cap H_{i+2}$, it is easy to see that $\ell(g)=2 d\left(H_{i}, H_{i+2}\right)$. Therefore injrad $\left(\Gamma^{+}(P)\right) \leq \ell(g) / 2=d\left(H, H^{\prime}\right)<\cosh ^{-1}(3+4 \cos (2 \pi / n))$. 


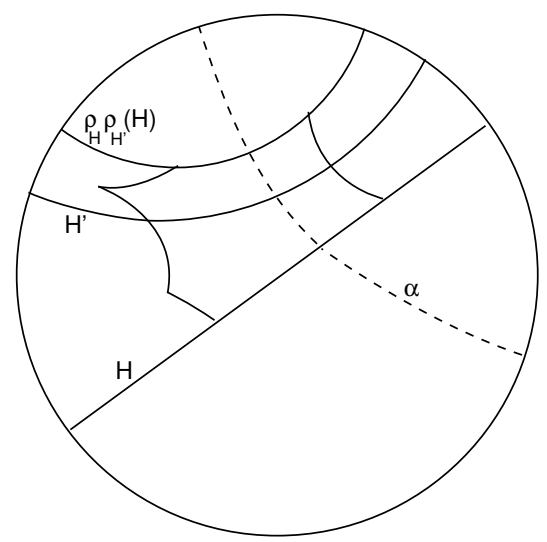

Figure 5. The distance from $H$ to $H \prime$ is half the translation length of $\rho_{H} \rho_{H /}$.

If $P$ is a triangle, suppose first that $P$ has no right angles, and is compact. Label the vertices of $P$ by $v_{0}, v_{1}, v_{2}$, with corresponding angles $\theta_{0}, \theta_{1}, \theta_{2}$ (see Fig. 6a). One of the angles, say $\theta_{0}$, must be $\leq \pi / 4$. Consider the quadrilateral $Q$, with angles $\left(\theta_{0}, 2 \theta_{1}, \theta_{0}, 2 \theta_{2}\right)$, obtained by reflecting $P$ along $v_{1} v_{2}$. Let $H$ and $H^{\prime}$ be non-adjacent bounding geodesics of $Q$, with $d\left(H, H^{\prime}\right)<\cosh ^{-1}(3+4 \cos (2 \pi / 4))$. $H$ and $H^{\prime}$ are disjoint: For if they intersected, they would create a triangle $T$ with angles $\left(\pi-\theta_{0}, \pi-2 \theta_{i}, x\right)$, for $\mathrm{i}=1$ or 2 ; however $\theta_{0}+2 \theta_{i}<\pi$, so $T$ would have angle sum $>\pi$, which is impossible. Therefore, as above, $g=\rho_{H} \rho_{H^{\prime}}$ is a hyperbolic element of $\Gamma^{+}(P)$ with $\ell(g) / 2<\cosh ^{-1}(3+4 \cos (2 \pi / 4))$.

If $P$ has a right angle at a vertex $v_{0}$, and is compact, reflect $\mathrm{P}$ along $v_{0} v_{1}$ to obtain a new triangle, $P^{\prime}$, with angles $\left(2 \theta_{1}, \theta_{2}, \theta_{2}\right)$ (see Fig. $\left.6 \mathrm{~b}\right)$. Reflect $P^{\prime}$ along $v_{0} v_{2}$ to obtain a quadrilateral $Q$ with angles $\left(2 \theta_{1}, 2 \theta_{2}, 2 \theta_{1}, 2 \theta_{2}\right)$. Then, again, the opposite geodesics bounding $Q$ must be disjoint, or else they would create a triangle with angles $\left(\pi-2 \theta_{1}, \pi-2 \theta_{2}, x\right)$, which is impossible since $\theta_{1}+\theta_{2}<\pi / 2$. So again we obtain a hyperbolic element $g$ with $\ell(g) / 2<$ $\cosh ^{-1}(3+4 \cos (2 \pi / 4))$.

If $P$ has an ideal vertex $v_{0}$, then reflecting $P$ along $v_{1} v_{2}$ creates a quadrilateral $Q$ whose bounding geodesics clearly cannot intersect, so that again we obtain the required hyperbolic element.

As a corollary of Theorem 4.2, we have:

Corollary 4.4. Let $P$ be a finite-volume Coxeter polyhedron in $\boldsymbol{H}^{3}$ with an $n$-sided prismatic face. Then injrad $\left(\Gamma^{+}(P)\right) \leq \cosh ^{-1}(3+4 \cos (2 \pi / n))$. 


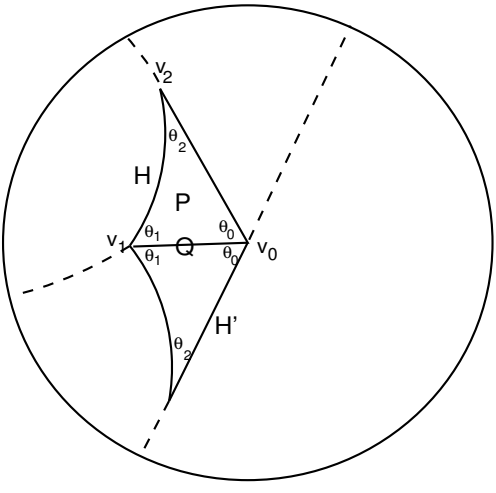

a

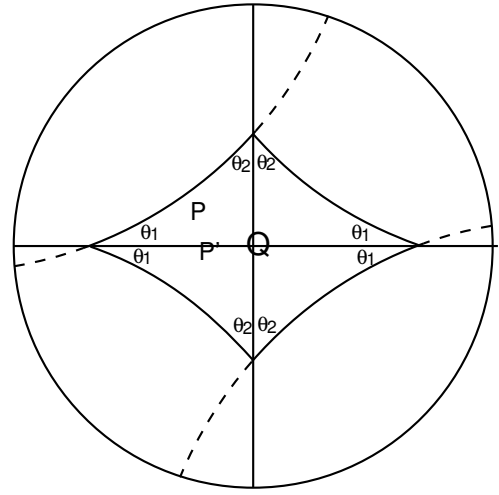

b

Figure 6. We can reflect to obtain a quadrilateral.

Proof. Consider the n-sided prismatic face $F_{0}$, and label the faces adjacent to $F_{0}$ cyclicly by $F_{1}, \ldots, F_{n}$. Let $H_{j}$ denote the hyperplane spanned by $F_{j}$, and let $H_{i, j}=H_{i} \cap H_{j}$. By Lemma 4.3, there is some i such that $d\left(H_{0, i}, H_{0, i+2}\right) \leq \cosh ^{-1}(3+4 \cos (2 \pi / n))$. Then $d\left(H_{i}, H_{i+2}\right) \leq \cosh ^{-1}(3+$ $4 \cos (2 \pi / n))$. Since $F_{i}$ and $F_{i+2}$ are non-adjacent, $H_{i}$ and $H_{i+2}$ do not intersect by $[\mathbf{A} \mathbf{1}]$. So $g=\rho_{H_{i}} \rho_{H_{i+2}}$ is a hyperbolic element of $\Gamma^{+}(P)$. So injrad $\left(\Gamma^{+}(P)\right) \leq \ell(g) / 2 \leq \cosh ^{-1}(3+4 \cos (2 \pi / n))$.

We now prove the Main Theorem.

Proof of 4.1 .

\section{Compact case.}

Case 1: $P$ has no triangular faces.

By Theorem 3.1, $P$ contains a prismatic quadrilateral or pentagonal face, and so by Corollary 4.4, we are done.

Case 2: $P$ has a triangular face, but $P$ is not a simplex.

Let $F_{0}$ be the triangular face, and label the faces adjacent to $F_{0}$ by $F_{1}, F_{2}$ and $F_{3}$. Let $P I=\bigcap_{i=0,1,2,3} H_{i}^{+}$, where $H_{i}$ denotes the hyperplane spanned by $F_{i}$ (recall Section 2 for the definition of $H_{i}^{+}$). Let $C_{i}$ and $C_{i}^{+}$denote the 
boundary at infinity of $H_{i}$ and $H_{i}^{+}$, respectively. Let $\theta_{i j}=$ dihedral angle between $F_{i}$ and $F_{j}=\pi / n_{i j}$ (here we are again using the fact that in the compact case the polyhedra are simple). We label the dihedral angles of $P$ ' by $\mathbf{A}_{P^{\prime}}=\left(\left(\mathrm{n}_{01}, \mathrm{n}_{02}, \mathrm{n}_{03}\right),\left(\mathrm{n}_{12}, \mathrm{n}_{23}, \mathrm{n}_{31}\right)\right)$. Then,

$$
\begin{array}{ll}
\text { I. } & 1 / n_{0 i}+1 / n_{0 j}+1 / n_{i j}>1, \quad \text { and } \\
\text { II. } & 1 / n_{12}+1 / n_{23}+1 / n_{31}<1 .
\end{array}
$$

One may then easily verify that the only 5 possibilities for $\left(n_{01}, n_{02}, n_{03}\right)$ are: $(2,2,2),(2,2,3),(2,2,4),(2,2,5)$ and $(2,3,3)$.

Case $2 \mathrm{a}: \mathbf{A}_{P^{\prime}}=\left((2,2,2),\left(n_{12}, n_{23}, n_{31}\right)\right)$ or $\left((2,2,4),\left(n_{12}, n_{23}, n_{31}\right)\right)$.

Let $\mathrm{Stab}_{F_{0}}$ denote the subgroup of $\Gamma^{+}(P)$ which leaves $F_{0}$ invariant. Then Theorem 5.4 of $[\mathbf{B M}]$ implies that Stab $_{F_{0}}$ contains a triangle group. So by Theorem 4.2, we are done.

Case $2 \mathrm{~b}: \mathbf{A}_{P \prime}=\left((2,3,3),\left(n_{12}, n_{23}, n_{31}\right)\right)$.

By I and II, the only two possibilities (modulo relabeling of edges) are $\mathbf{A}_{P^{\prime}}=((2,3,3),(4,2,5))$ or $((2,3,3),(5,2,5))$. Consider $P \prime \prime=P \prime \cup \rho_{H_{1}}(P \prime)$ (see Fig. 7a). Let $Q$ denote the quadrilateral created in $H_{0}$. Conjugate so that, in the upper half space model, $C_{1}$ is the imaginary axis (see Fig. 7b), and $\infty \in C_{i}^{+}-C_{i}$ for $\mathrm{i}=2,3 . H_{1} \cap H_{2} \cap H_{3}=\emptyset$, since the angles $\theta_{0 i}$ are acute; therefore, $C_{2}^{+} \cap C_{3}^{+} \cap \rho_{H_{1}}\left(C_{2}^{+}\right) \cap \rho_{H_{1}}\left(C_{3}^{+}\right)=Q_{\infty}^{1} \cup Q_{\infty}^{2}$, where $Q_{\infty}^{1}, Q_{\infty}^{2}$ are quadrilateral regions in $S_{\infty}^{2}=\hat{\mathbb{C}}$, and $\infty \in Q_{\infty}^{2}$. The dihedral angles of $Q_{\infty}^{1}$ are $\left(\theta_{23}, 2 \theta_{12}, \theta_{23}, 2 \theta_{31}\right)$.

We claim that $C_{2} \cap \rho_{H_{1}}\left(C_{3}\right)=C_{3} \cap \rho_{H_{1}}\left(C_{2}\right)=\emptyset$, in which case $H_{2}, \rho_{H_{1}}\left(H_{3}\right)$ are disjoint, and $H_{3}, \rho_{H_{1}}\left(H_{2}\right)$ are disjoint. Otherwise, three circles would intersect to create a triangle $T$ with angle sum $>\pi$ (see Fig. $7 \mathrm{c}$ ). However, $T$ has only one positively curved side - call its curvature $\kappa$ - and it has another, longer side of curvature $-\kappa$. So, by the Gauss-Bonnet formula, $T$ has angle sum $<\pi$, for a contradiction. Hence $g_{1}=\rho_{H_{2}} \rho_{\rho_{H_{1}}\left(H_{3}\right)}$ and $g_{2}=\rho_{H_{3}} \rho_{\rho_{H_{1}}\left(H_{2}\right)}$ are both hyperbolic elements of $\Gamma^{+}(P)$. By Lemma 4.3, two of the opposite faces of $Q$ must be less than $\cosh ^{-1}(3+4 \cos (2 \pi / 4))$ apart. It follows that either $g_{1}$ or $g_{2}$ has suitably short translation length.

Case 2c: $\mathbf{A}_{P \prime}=\left((2,2,3),\left(n_{12}, n_{23}, n_{31}\right)\right)$ or $\left((2,2,5),\left(n_{12}, n_{23}, n_{31}\right)\right)$.

Without loss of generality, assume $n_{13} \geq n_{23}$, so by II, $n_{13} \geq 3$. First suppose $n_{12}, n_{23} \geq 3$. As in Case $2 \mathrm{~b}$, reflect in $H_{1}$ to create a polyhedron $P \prime \prime$ with a quadrilateral face $Q$. Again, on $S_{\infty}^{2}$ we see a quadrilateral $Q_{\infty}^{1}$ with 

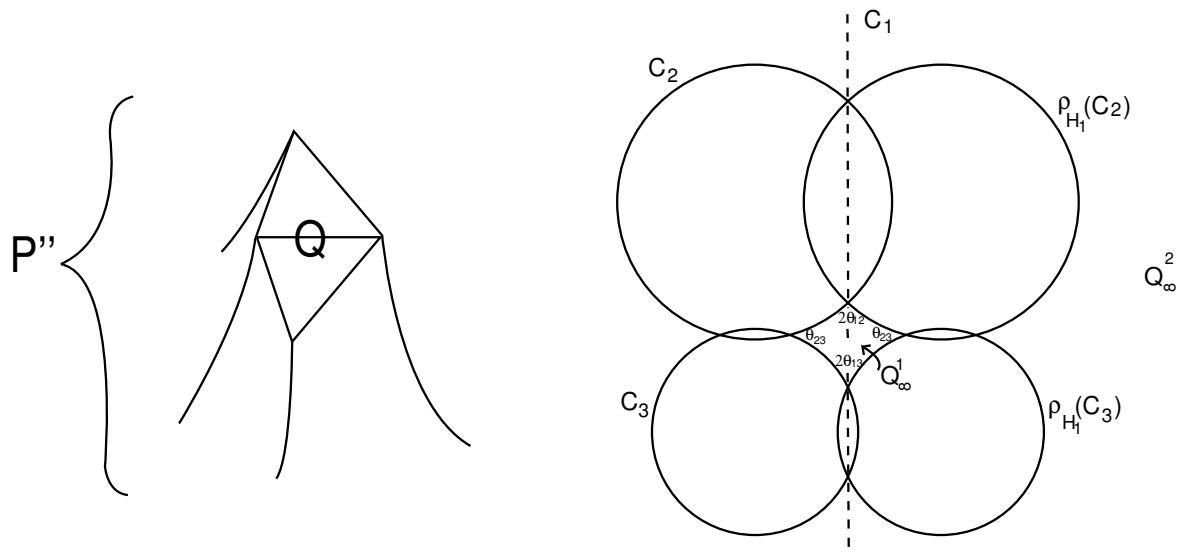

a

b

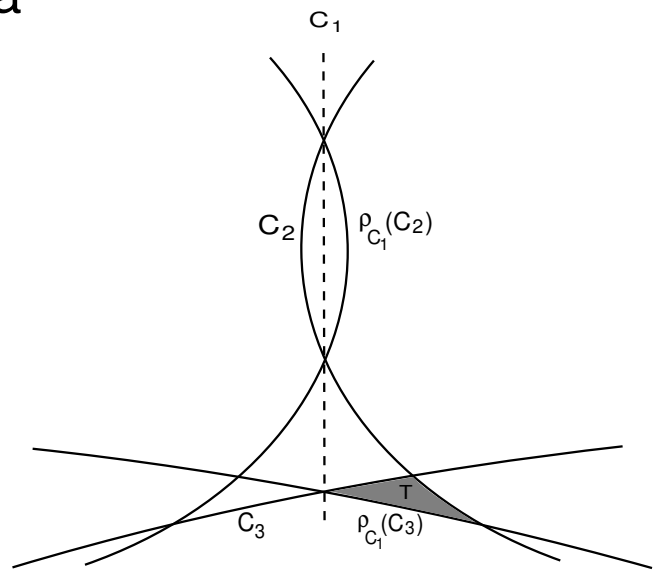

C

Figure 7. a) The polyhedron $P \prime \prime$. b) The view on the sphere at infinity. c) If opposite circles intersect, a triangle is formed.

angles $\left(\theta_{23}, 2 \theta_{12}, \theta_{23}, 2 \theta_{31}\right)$. Since the sum of any two adjacent angles of $Q_{\infty}^{1}$ is $\leq \pi$, we can argue as in Case $2 \mathrm{~b}$ to show that opposite circles bounding $Q^{\prime}$ must be disjoint, thus creating a hyperbolic element with suitably short translation length.

If $n_{12}=2$, then by I and II, $n_{23}$ and $n_{31} \geq 4$. Then after reflecting in $H_{1}$ and in $H_{2}$, we see on $S_{\infty}^{2}$ an acute quadrilateral (see Fig. 8), and we may argue as above.

If $n_{23}=2$, then by I and II, $n_{12} \geq 4$. 

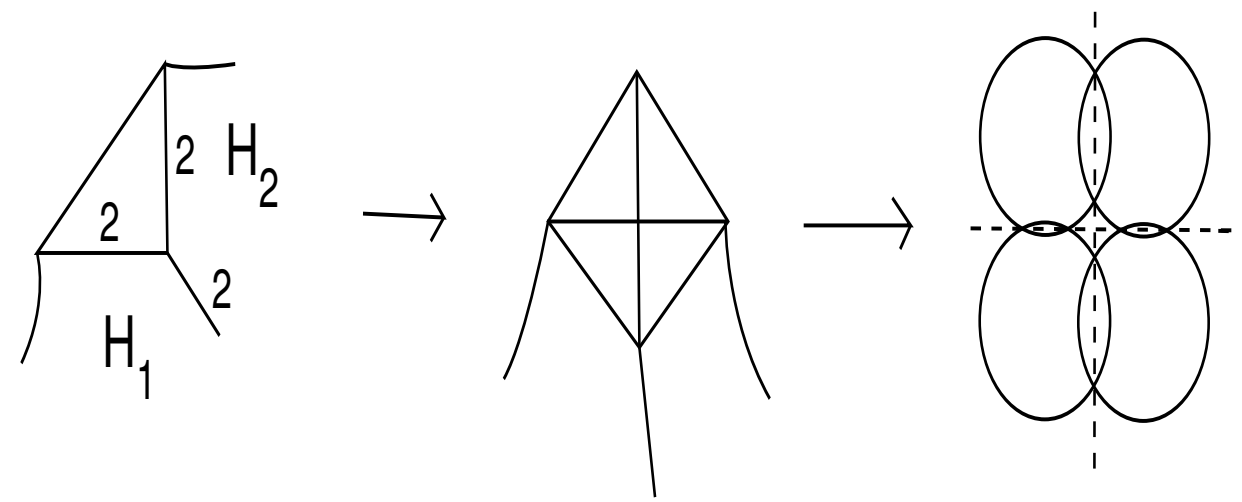

Figure 8. Reflect twice, then view at infinity.

- If $n_{12}=4$, then after reflecting in $H_{2}$, we can reduce to the case where $n_{12}=2$.

- If $n_{12} \geq 5$, then by II, $n_{23} \geq 4$, and reflecting in $H_{2}$ creates an acute quadrilateral. So we may argue as in Case $2 b$.

Case 3: $\mathrm{P}$ is a simplex.

By $[\mathbf{L}]$ there are only nine congruence classes of compact simplices in $\mathbf{H}^{3}$. By $[\mathbf{M}]$ (see also $[\mathbf{B M}]$ ), eight of these contain triangle groups, so in these cases the result follows from Theorem 4.2. Denote the remaining tetrahedron by $T_{8}$; label its faces $F_{1}, \ldots, F_{4}$; and let $\pi / n_{i j}$ be the dihedral angle between $F_{i}$ and $F_{j}$. We have $n_{12}=2, n_{13}=3, n_{14}=4, n_{23}=5, n_{24}=3$, and $n_{34}=4$. Let $H_{i}$ be the hyperplane spanned by $F_{i}$. It is not difficult to construct $T_{8}$ explicitly in Lobachevsky space and then compute the faithful discrete representation of $\Gamma^{+}\left(T_{8}\right)$ in $O(3,1)$. Then it is straightforward to compute that $\rho_{H_{3}} \rho_{H_{4}} \rho_{H_{2}} \rho_{H_{1}} \rho_{H_{4}} \rho_{H_{2}}$ is a hyperbolic element with translation length $1.66131 \ldots<2 \cosh ^{-1}(3+4 \cos (\pi / 5))$.

\section{Non-compact case:}

By Theorem 3.1 and Corollary 4.4, it is enough to consider the case where $P$ has a triangular face, $F_{0}$. As in the compact case, the faces adjacent to $F_{0}$ form a polyhedron $P \prime$.

If none of the vertices of $F_{0}$ are ideal, then the proof for the compact case carries over without change. So suppose $F_{0}$ has an ideal vertex.

Ideal vertices may be either tri-valent or 4 -valent (see [A2]). If the vertices of $F_{0}$ are all trivalent then condition II still holds; condition I holds at regular 
vertices, and changes to an equality at ideal vertices. Then the techniques from the compact case are sufficient to prove the theorem- we omit the details. If one of the vertices is 4 -valent, then two of the sides adjacent to $F_{0}$ are tangent on $S_{\infty}^{2}$ (see Fig. 9). One may now argue as in the compact case, treating the tangent sides as adjacent with dihedral angle 0 . The theorem follows.

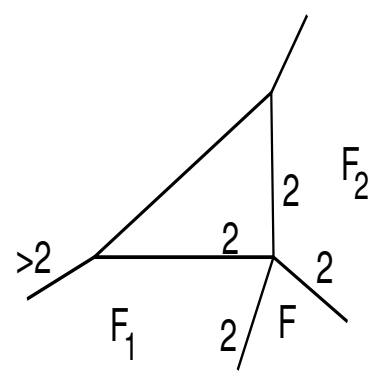

a

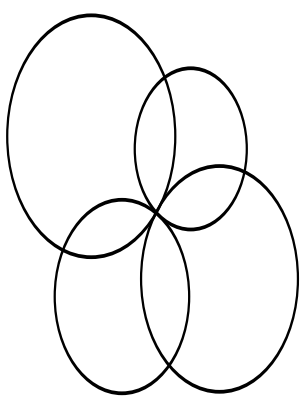

b

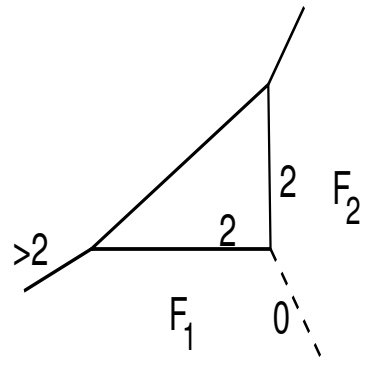

C

Figure 9. a) A triangular region with a 4-valent ideal vertex. b) A 4 -valent ideal vertex on $S_{\infty}^{2}$. c) We may remove $F$ and view $F_{1}$ and $F_{2}$ as adjacent with dihedral angle 0 .

\section{References}

[AR] C.C. Adams and A.W. Reid, Systoles for hyperbolic knot and link complements, Math. Proc. Camb. Phil. Soc., to appear.

[A1] E.M. Andreev, On convex polyhedra in Lobačevskï spaces, Math. USSR-Sb., 10 (1970), 413-440.

[A2] - On convex polyhedra of finite volume in Lobačevskii spaces, Math. USSRSb., 12 (1970), No. 2, 255-259.

[BM] T. Baskan and A.M. Macbeath, Centralizers of reflections in crystallographic groups, Math. Proc. Camb. Phil. Soc., 92 (1982), 79-91.

[B] K. Brown, Buildings, Springer-Verlag, New York, 1989.

[L] F. Lanner, On complexes with transitive groups of automorphisms, Comm. Sem. Math. Univ. Lund, 11 (1950), 1-71.

[M] C. Maclachlan, Triangle subgroups of hyperbolic tetrahedral groups, Pacific J. Math., 176 (1996), 195-203.

[N] V.V. Nikulin, On arithmetic groups generated by reflections in Lobačevskĭ spaces, Math. USSR Izv., 16 (1981), 573-601. 
[V] E.B. Vinberg, Hyperbolic reflection groups, Russian Math. Surveys, 40 (1985), 3175 .

[W] M. White, preprint.

Received September 17, 1997 and revised March 7, 2000.

Department of Mathematics

UNIVERSITY OF TEXAS

Austin, TX 78712

Department of Mathematics

MS 136, RicE UNIVERSity

Houston TX 77005-1892

E-mail address: mastersj@rice.edu 